\title{
Single-crystal diffractometer coupled with double-sided laser heating system at the Extreme Conditions Beamline P02.2 at PETRAIII
}

Cite as: Rev. Sci. Instrum. 90, 073907 (2019); https://doi.org/10.1063/1.5108881

Submitted: 03 May 2019. Accepted: 10 July 2019 . Published Online: 31 July 2019

Elena Bykova (D), Georgios Aprilis (D), Maxim Bykov (D), Konstantin Clazyrin, Mario Wendt, Sergej Wenz, Hanns-Peter Liermann (D), Jan Torben Roeh (D), Anita Ehnes, Natalia Dubrovinskaia (D), and Leonid Dubrovinsky (i)

\section{ARTICLES YOU MAY BE INTERESTED IN}

Novel piezoelectric rotary motor driven by a single-phase sine wave with an asymmetric stator

Review of Scientific Instruments 90, 075006 (2019); https://doi.org/10.1063/1.5094798

Evaluation of a photoacoustic bone-conduction vibration system

Review of Scientific Instruments 90, 074905 (2019); https://doi.org/10.1063/1.5081078

Design of coaxial coupled structure for distance-insensitive wireless power transfer Review of Scientific Instruments 90, 074708 (2019); https://doi.org/10.1063/1.5095210

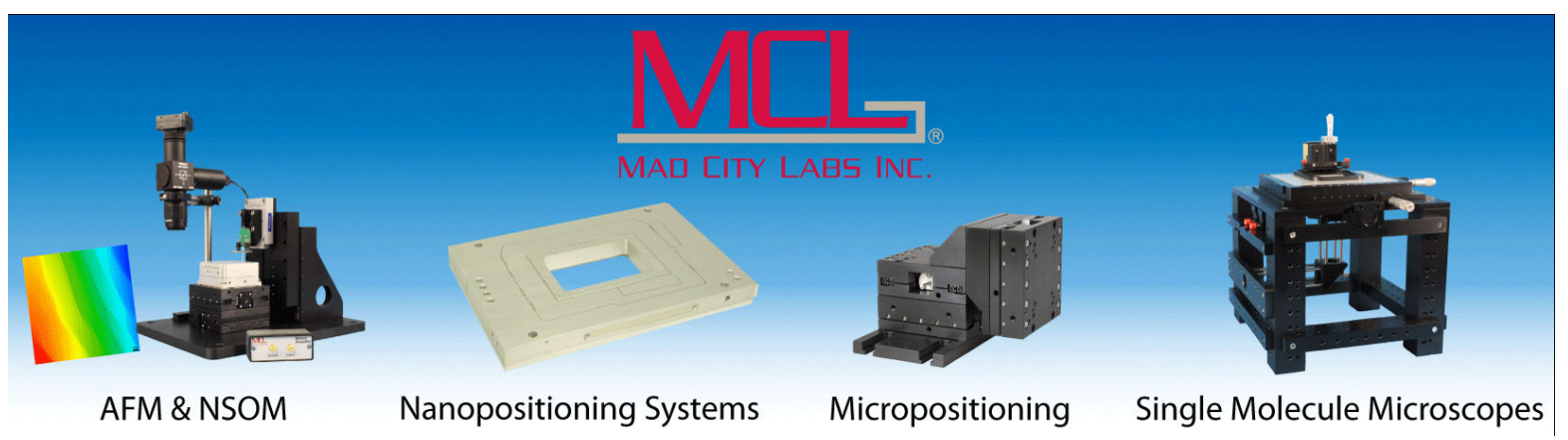




\title{
Single-crystal diffractometer coupled with double-sided laser heating system at the Extreme Conditions Beamline P02.2 at PETRAIII
}

\author{
Cite as: Rev. Sci. Instrum. 90, 073907 (2019); doi: 10.1063/1.5108881 \\ Submitted: 3 May 2019 Accepted: 10 July 2019 • \\ Published Online: 31 July 2019
}

\begin{abstract}
Elena Bykova, ${ }^{1, a)}$ (D) Georgios Aprilis, ${ }^{2}$ (D) Maxim Bykov, ${ }^{3}$ (D) Konstantin Glazyrin, ${ }^{2}$ Mario Wendt, Sergej Wenz, ${ }^{7}$ Hanns-Peter Liermann, ${ }^{1}$ (D) Jan Torben Roeh, ${ }^{4}$ (D) Anita Ehnes, Natalia Dubrovinskaia, ${ }^{2}$ (D) and Leonid Dubrovinsky ${ }^{3}$

\author{
AFFILIATIONS \\ ${ }^{1}$ Photon Science, Deutsches Elektronen-Synchrotron (DESY), Notkestrasse 85, Hamburg 22607, Germany \\ ${ }^{2}$ Material Physics and Technology at Extreme Conditions, Laboratory of Crystallography, University of Bayreuth, \\ Universitaetsstrasse 30, Bayreuth 95440, Germany \\ ${ }^{3}$ Bavarian Research Institute of Experimental Geochemistry and Geophysics, University of Bayreuth, \\ Universitaetsstrasse 30, Bayreuth 95440, Germany \\ ${ }^{4}$ Sample Environment and Extreme Conditions Science Infrastructure (ECSI), \\ Deutsches Elektronen-Synchrotron (DESY), Notkestrasse 85, Hamburg 22607, Germany
}

a) Author to whom correspondence should be addressed: elena.bykova@desy.de

\begin{abstract}
Combination of in situ laser heating with single-crystal X-ray diffraction (scXRD) in diamond anvil cells (DACs) provides a tool to study crystal structures and/or chemistry of materials at simultaneous high pressures and high temperatures. Here, we describe the first dedicated single-crystal X-ray diffractometer coupled with double-sided laser heating (dsLH) system. The scXRD/dsLH setup was developed for the P02.2 Extreme Conditions Beamline at PETRA III and became available for general users in 2017. It enables the collection of reliable scXRD data at simultaneous high pressure and high temperature. We demonstrate the performance of the setup on example of studies of iron and chromium nitrides.
\end{abstract}

Published under license by AIP Publishing. https://doi.org/10.1063/1.5108881

\section{INTRODUCTION}

Diamond anvil cell technique provides a tool to generate pressures, which can exceed those in the center of the Earth (360 GPa), and to collect in situ spectroscopy (Raman, Mössbauer, X-ray absorption, etc.) and/or X-ray diffraction data. However, the analysis and interpretation of the data obtained at multimegabar pressures is extremely difficult, especially due to multiple phase transitions and/or chemical reactions, formation of unusual crystal structures and/or chemical compounds unknown at ambient conditions.

Single-crystal X-ray diffraction (scXRD) in DACs opens a way to determine not only the crystal structure of a material but also to refine its chemical composition for even very complex multiphase and/or multigrain samples. Due to significant progress in the field of high-pressure technology, since a decade scXRD became available at pressures above $\sim 15 \mathrm{GPa}$. Novel gas-loading systems ${ }^{1-6}$ allow one to use soft pressure transmitting media (helium or neon) in DACs, thus preserving single crystals to megabar pressures. New types of diamond anvils and DACs with wide X-ray opening up to $90^{\circ 7}$ enlarge the coverage of the reciprocal space and thus improve the data redundancy which leads to the higher quality of the final structural models.

Heating is an important parameter for high-pressure experiments, especially for studies dedicated to modeling of processes in 
deep Earth's interior. Double-sided laser-heating (dsLH) systems are widely used at synchrotron facilities..$^{8-11}$ However, until recently, all of them were stationary and did not allow DAC rotation during simultaneous heating, thus making in situ high-pressure hightemperature scXRD impossible. A portable laser-heating system, first developed in Bayerisches Geoinstitut (BGI), ${ }^{12}$ can be mounted directly on a goniometer stage and rotated along with the DAC, providing the opportunity to collect scXRD simultaneously during laser heating.

Here, we describe the first dedicated scXRD/dsLH setup consisting of a single-crystal X-ray diffractometer coupled with doublesided laser heating system. The setup was developed for beamline P02.2, the Extreme Conditions Beamline (ECB), at PETRA III and became available for the general users in 2017.

\section{SETUP OVERVIEW}

The scXRD/dsLH setup is mounted on the General Purpose experiment of beamline P02.2 ${ }^{13}$ (Fig. 1). The major components of the setup (from the top to the bottom) are listed below and shown in Fig. 2:

- sample holder equipped with water cooling;

- two portable universal laser heating heads (UniHeads, Precitec ${ }^{\mathrm{TM}}$ ) equipped with $4 \mathrm{~mm}$-thick glassy carbon mirrors; one UniHead is mounted horizontally (the upstream h-UniHead), the other one, vertically (the downstream v-UniHead); and

- multiaxis positioning systems realized through the combination of translation positioning stages:

- motorized Newport X- and Y-stages and motorized Huber Z-stage for DAC alignment;

- motorized Huber XY- and Huber Z-stages for upstream h-UniHead; and
- manual Standa Z-stage for downstream v-UniHead;

- stabilization elements (aluminum frame with steel pillars and supporting corner elements, clamp for h-Unihead, counterbalance for the dsLH system, etc.) to decrease vibrations and improve the stability of heating during rotation; and

- 4-axis positioning system for alignment of dsLH system composed of the following translation stages:

- Huber motorized XY-stage;

- rotational positioning device, 2-circle $(\omega-2 \theta)$ Huber goniometer; and

- heavy-load Huber motorized Z-stage;

- heavy-load Huber motorized XY-stages for the alignment of the $\omega$ rotation axis to the focus of the X-ray beam;

- aluminum base plate (50 $\mathrm{mm}$ thick) integrating the entire setup, including an X-ray diode and a beamstop; and

- additional components of the setup, which are not shown in Fig. 2:

- two SPI RedPower R4 fiber lasers (50 W, excitation wavelength of $1070 \mathrm{~nm}$ );

- two Ocean Optics QE65000 spectrometers for collection of the thermal radiation; and

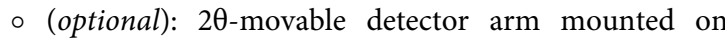
$\omega$-stage (for Lambda or Pilatus detectors).

The scheme of the optical components in the dsLH system is adopted from Kupenko et al. ${ }^{12}$ (see Fig. 3). Two UniHeads ${ }^{12}$ are used to focus the laser beams on the sample from both the sides (Figs. 2 and 3). Each of them includes a compact set of optical components. The $90^{\circ}$ arrangement of the two UniHeads serves to minimize the shadows cast on the detector by various components of the system to reduce possible vibrations of the optical components and to achieve the largest possible $\omega$ rotation range. The glassy carbon

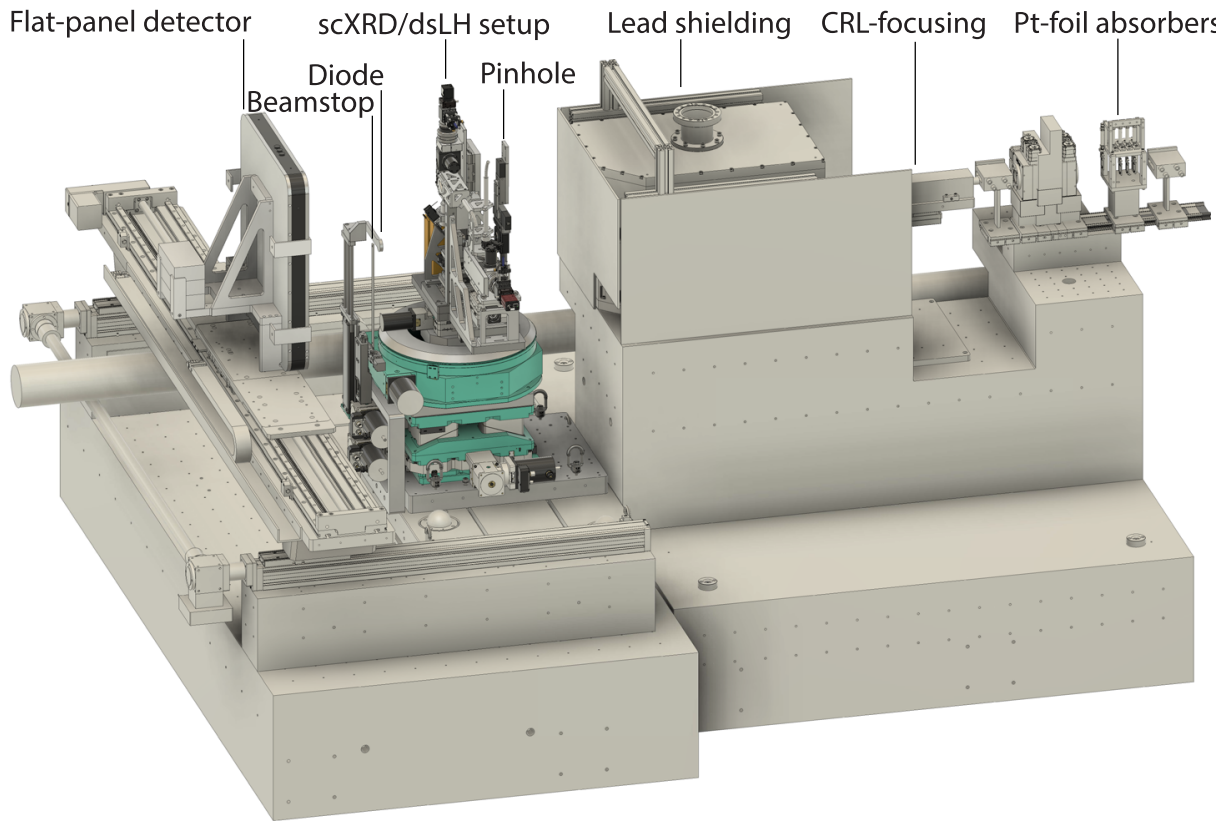

FIG. 1. Overview of the $s c X R D / d s L H$ setup mounted on the General Purpose experiment at PETRAIll beamline P02.2 (ECB). The primary X-ray beam passes through the set of Compound Refractive Lenses (CRLs) focusing the beam down to $\sim 5 \times 5 \mu \mathrm{m}^{2}$. Lead shielding covers X-ray optics in order to reduce parasitic scattering. During rotation of the scXRD/dsLH setup as a whole, diffraction data are collected using a Perkin Elmer XRD1621 flat panel detector. To allow rotation, the X-ray pinhole is located at a distance of about $80 \mathrm{~mm}$ from the sample. The intensity monitor (diode) and the beamstop are attached to the mounting plate of the $s c X R D / d s L H$ setup. Platinum-foil (Pt-foil) absorbers can be optionally used to attenuate the primary X-ray beam intensity. 
(a)

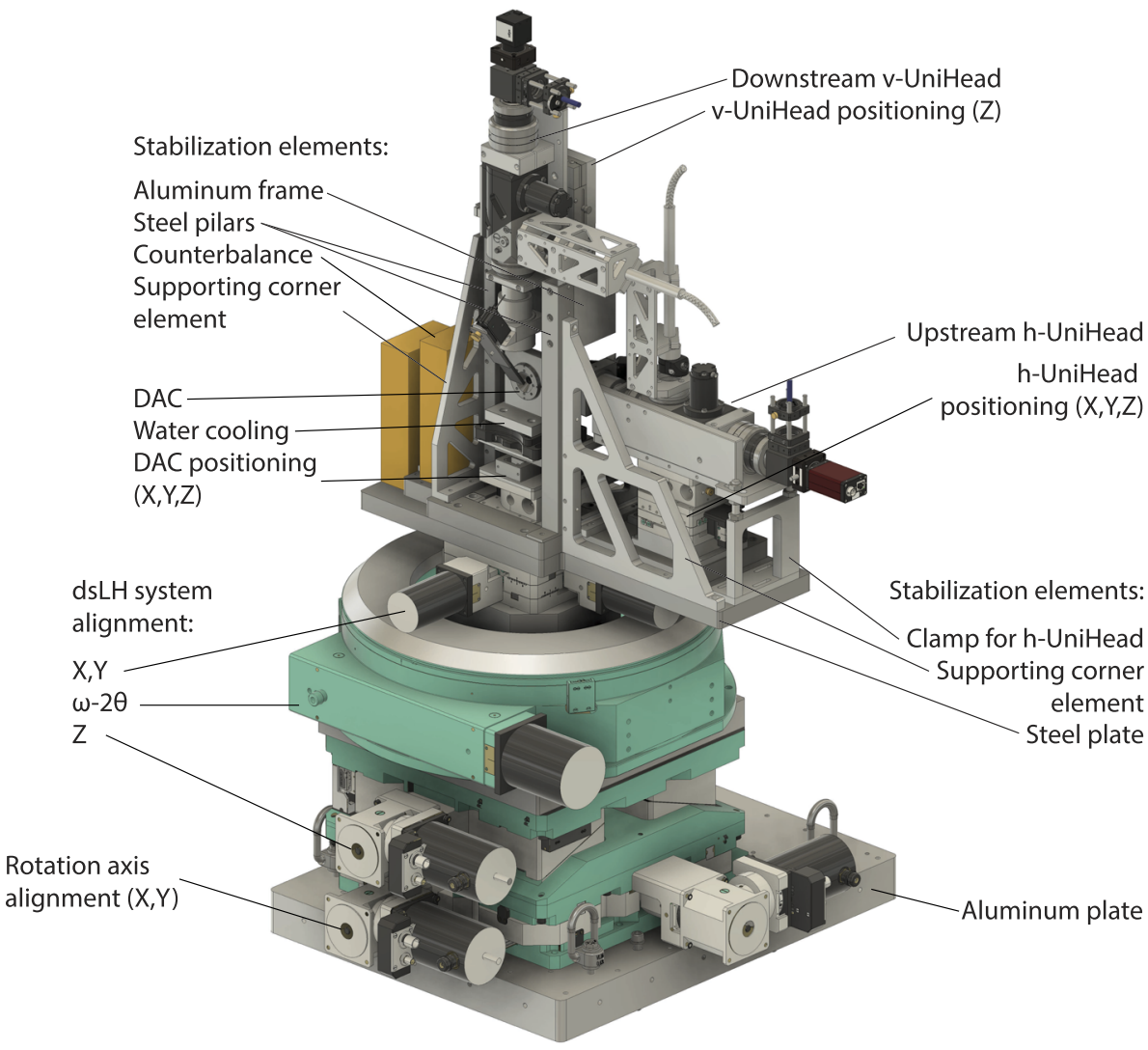

FIG. 2. ScXRD/dsLH setup: view from the downstream side (a); from the upstream side (b); and perpendicular to the X-ray beam (c).

(b)
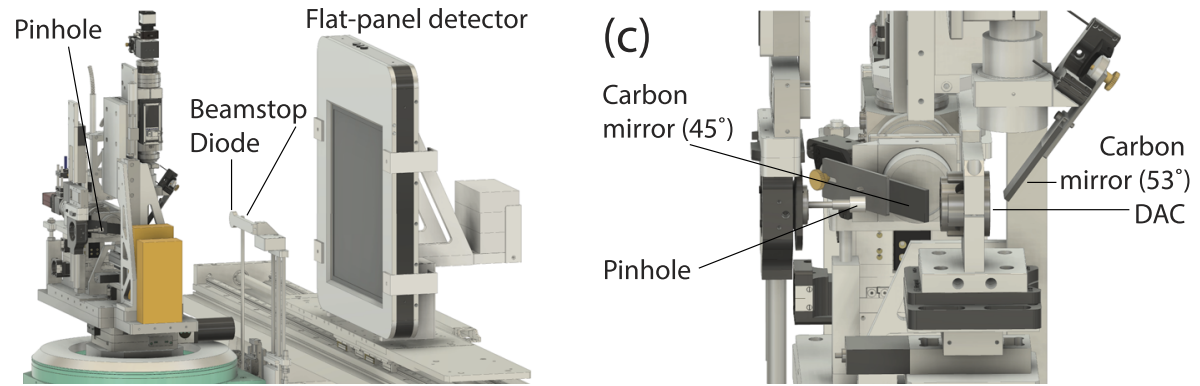

mirrors are mounted at an angle to the axes of the UniHeads [Figs. 2(c) and 3] and serve as a reflector of the laser light in order to direct it to the sample. The upstream mirror is mounted precisely at $45^{\circ}$, which aligns the laser beam parallel (coaxial) to the X-rays that penetrate the mirror. The downstream mirror is placed at a larger angle, between $50^{\circ}$ and $55^{\circ}$, to avoid primary X-ray beam interaction and prevent strong unwanted diffuse scattering. Both the mirror holders are connected to the Newport piezo mounts for fine tuning of the final laser beam position.

For the sample illumination during alignment, the UniHeads are equipped with built-in light emitting diodes (LEDs) controlled externally. In the system, the sample is visualized using $\mu \mathrm{EYE}$ (downstream) and Manta G-125 (upstream) CCD cameras equipped with IR-filters. Laser focusing and sample observation use the same optical path. We installed GeoHEAT 40_NIR objective (AdlOptica $\mathrm{GmbH}$ ) that provides a working distance of $80 \mathrm{~mm}$ at a focal length of $40.8 \mathrm{~mm} .{ }^{14}$ A long working distance is necessary to keep optics away from the primary beam, yet a short focal length provides higher magnification, which in this case is $20 \times$ for the system in total.

The SPI fiber lasers used for sample heating are capable to operate in continuous and modulated modes with a maximum modulation frequency of $100 \mathrm{kHz}$. Each fiber laser is equipped with a pilot laser, which emits light in the range of $630-680 \mathrm{~nm}$ and may be used for prealignment. Electrically tunable lenses (Optotune ${ }^{\mathrm{TM}}$ ) are used for focusing of the laser spots; they are installed directly on the end of the laser fibers. Laser fibers are mounted on the UniHeads with lightweight aluminum holders designed and 


\section{Downstream Upstream}

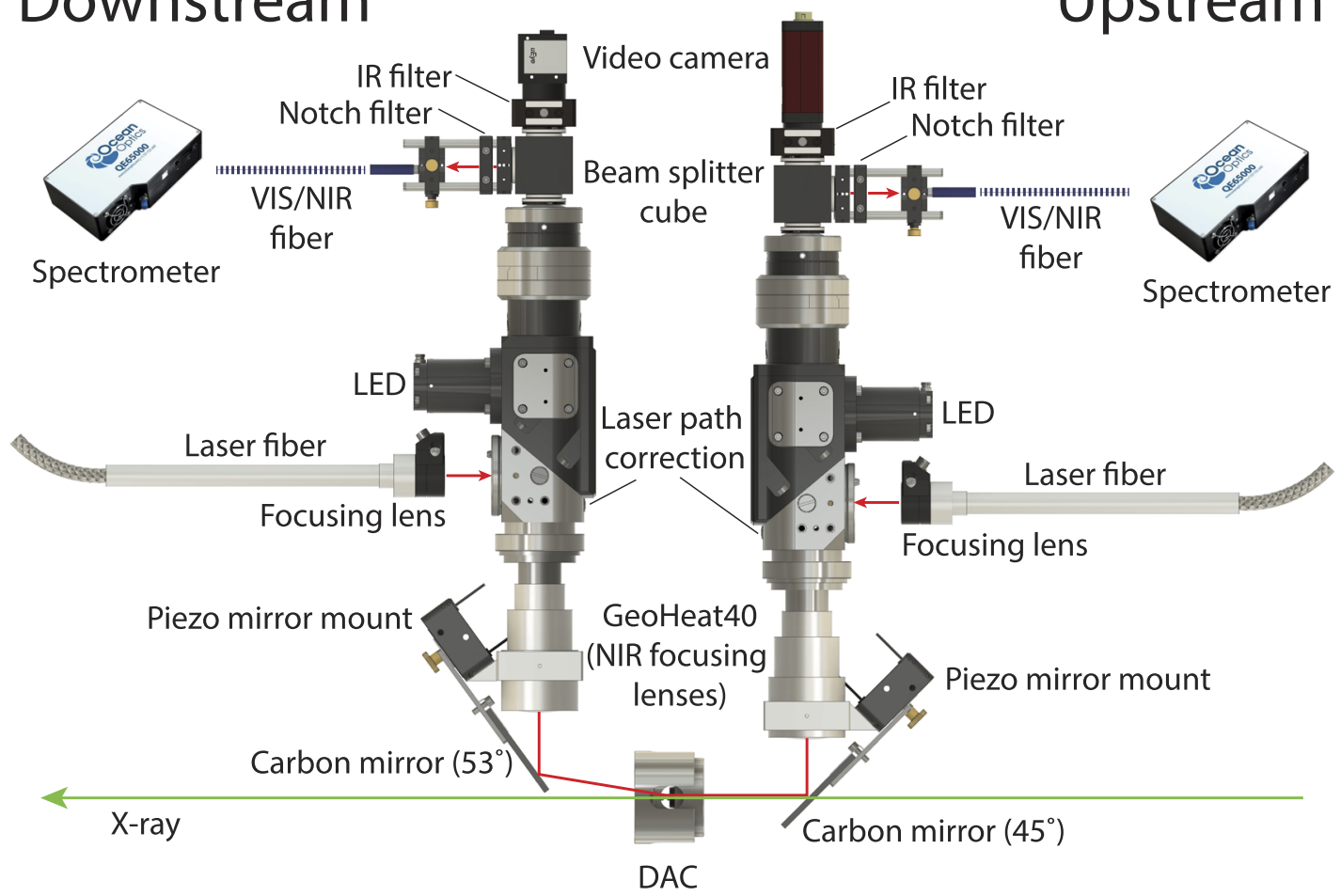

FIG. 3. Schematic diagram of the optical components of the dsLH system (based on the system described in Ref. 12). The UniHeads are aligned mutually perpendicular.

constructed specifically to reduce vibrations of the laser beam during $\omega$-rotation.

The scXRD/dsLH setup contains all the components required for data collection and laser heating in one module. It can be mounted/unmounted on the General Purpose experiment at P02.2 (Fig. 1) within a few hours.

\section{SETUP OPERATION}

\section{A. Preparation of the beamline}

In comparison with the standard sample stack at the General Purpose experiment, the scXRD/dsLH setup demands additional space between the sample and the lead shielding (which encapsulates X-ray optics) to allow for its rotation (Fig. 1). This requires a longer X-ray focusing distance. The focusing distance of $1477 \mathrm{~mm}$ is achieved by using 91 Compound Refractive Lenses (CRLs) at an $\mathrm{X}$-ray energy of approximately $42.7 \mathrm{keV}$ (Fig. 1). The position of the $\mathrm{X}$-ray focus is modified by moving either the CRLs or the heavyload Huber motorized X-stage along the beam direction. A standard tungsten round edge is used to estimate the focus size (typically $\sim 5$ $\times 5 \mu \mathrm{m}^{2}$ ). A thin tungsten cross hair is used to calibrate the beam position and bring the rotation $\omega$-axis to the beam by using the heavy-load Huber motorized Y- and Z-stages.

The sample-to-detector distance, coordinates of the beam center, and tilt angle and tilt plane rotation angle of the detector images are calibrated using $\mathrm{CeO}_{2}$ standard powder. In addition, a single crystal of orthoenstatite $\left[\left(\mathrm{Mg}_{1.93}, \mathrm{Fe}_{0.06}\right)\left(\mathrm{Si}_{1.93}, \mathrm{Al}_{0.06}\right) \mathrm{O}_{6}, P b c a, a=\right.$ 8.8117(2), $b=5.18320(10)$, and $c=18.2391(3) \AA]$ is used to calibrate the instrument model of the CrysAlisPro ${ }^{\circledR}$ software (sampleto-detector distance, the detector's origin, offsets of the goniometer angles, and rotation of the X-ray beam and the detector around the instrument axis).

\section{B. Alignment of the lasers and temperature measurements}

The initial alignment of the lasers is accomplished via a piece of platinum foil by centering both guiding lasers on the observation area using adjusting screws on the UniHeads. The final adjustments are made using the Pt thermal glow appearing when a sufficient near-infrared radiation (NIR) laser power is achieved.

Thermal radiation from both sides of the sample is collected using two optical fibers with a core diameter of $400 \mu \mathrm{m}$, which are connected to the Ocean Optics QE65000 spectrometers. Each optical fiber is aligned to the heating spot and focused on the image plane. To do so, the end of the optical fiber leading to the spectrometer is connected to a strong visible light source which illuminates the $\mathrm{Pt}$ foil. The position of the light spot on the foil is then adjusted by moving the screws which mount the optical fiber to the UniHead. In this way, due to the magnification of the system, the sample temperature is probed from an area of $20 \mu \mathrm{m}$ diameter, centered at the heated spot.

The thermal radiation spectra are analyzed using spectroradiometry $^{15}$ and fitted using the gray body approximation of 
Planck's law in the wavelength range of $650-800 \mathrm{~nm}$ with software developed for this purpose. Temperatures are calibrated with tungsten halogen lamp (OPTEEMA Engineering GmbH, OL-245M-K3) placed on the sample stage and precalibrated at 2200, 2500, and $2900 \mathrm{~K}$.

For temperature measurements during the $\omega$ rotation of the sample, thermal spectra are collected every second with exposure time of a few hundred milliseconds. The stage rotation and the temperature data collection of the detector are synchronized manually. The setup allows maintaining the laser heated sample under stable temperature during the entire data collection with a temporal variation that is well below the typical temperature measurement uncertainty of $\pm 100 \mathrm{~K}$ (Fig. 4).

\section{Installation of a DAC}

After a DAC is placed in the holder, the sample alignment is accomplished in three steps. In the first step, the upstream and downstream lasers are aligned to the center of the gasket. Using a video camera and the motorized $\mathrm{X}-$, $\mathrm{Y}$-, and $\mathrm{Z}$-stages right below the DAC, the sample is first positioned from the downstream vUniHead observation side. Then, the upstream h-UniHead is moved into position using the motorized XY- and Z-stages below it. In the second step, using the motorized XY-stage, the heavy-load Z-stage and the $\omega-2 \theta$ goniometer below the dsLH system, the DAC together with the aligned lasers is moved into the X-ray beam as well as to the center of the rotation axis using the standard procedure of the sample centering by X-ray absorption. ${ }^{16}$ Finally, the exact point of interest on the sample can be chosen by moving the X-, Y-, and $\mathrm{Z}$-stages below the DAC while the sample is observed through the video camera. Currently, the motors allow the DAC movement by

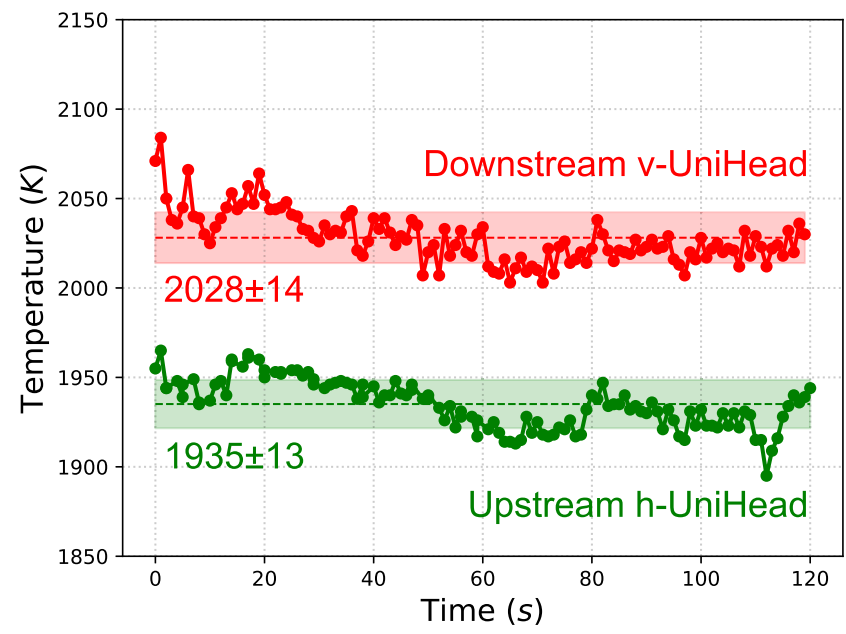

FIG. 4. Example of time-dependence of the temperature during laser heating and simultaneous scXRD data collection (step-scan) recorded from both sides of the heated sample of $\mathrm{CrN}$; green dots designate the upstream side temperature and red dots designate the downstream side temperature. Thermal radiation is collected every second with an exposure time of $800 \mathrm{~ms}$. Solid lines connecting the dots are guides for the eye. Dashed lines show the mean temperature values for each side over the entire rotation. Shaded areas represent the margins of one standard deviation. $\pm 25 \mu \mathrm{m}$ with an accuracy of $0.003 \mu \mathrm{m}$ in horizontal (X and $\mathrm{Y}$ ) directions, while in the vertical $(\mathrm{Z})$ direction, it is possible to move by $\pm 15 \mu \mathrm{m}$ with an accuracy of $0.003 \mu \mathrm{m}$.

The scXRD/dsLH setup provides a possibility to work with various types of DACs. However, the appropriate thickness of a DAC (including a membrane cup, if used) is limited to $50 \mathrm{~mm}$ to enable correct positioning of the mirrors.

\section{Data collection}

The scXRD data collection is realized in an oscillation (=narrow slicing) mode, i.e., when the diffraction is recorded while rotating the sample about a single $\omega$-axis in small steps of $0.5^{\circ}-1^{\circ}$. During the data collection combined with the laser heating, it is possible to collect the frames in ranges of $\omega$ from $-38^{\circ}$ to $+25^{\circ}$. Diffraction data are measured using a Perkin Elmer XRD1621 flat panel detector in a shutterless mode that reduces the data collection time to 1 or $2 \mathrm{~min}$ for the entire $\omega$ rotation range. Platinum-foil (Pt-foil) absorbers of the beamline may be used to decrease the intensity of the primary beam in order to avoid oversaturated diffraction peaks on the detector.

\section{E. Known problems and limitations}

At the moment, the scXRD/dsLH setup for X-ray diffraction experiments provides an even power distribution within the laser beam with a diameter of $10-30 \mu \mathrm{m}$, since the size of the homogeneously heated area should be significantly larger than the diameter of the X-ray beam of $\sim 5 \times 5 \mu \mathrm{m}^{2}$. Rotation of a DAC results in vibration of the sample around the aligned position with a magnitude of about $1-2 \mu \mathrm{m}$ during the entire data collection (from $-38^{\circ}$ to $+25^{\circ}$ ). In experiments up to about $100 \mathrm{GPa}$, in which the size of a sample is of about $10 \mu \mathrm{m}$, such vibrations are acceptable, as the sample still remains irradiated. However, in experiments above $100 \mathrm{GPa}$, the size of a sample drastically decreases and the size of the laser beam should be reduced down to $1-10 \mu \mathrm{m}$. This might require significant modifications of optical schemes and components as well as mechanics of the system to improve the stability further.

\section{EXAMPLES OF THE SETUP APPLICATION}

We have conducted a number of experiments to demonstrate capabilities of the scXRD/dsLH setup for in situ studies of materials, during which high pressures and high temperatures are applied simultaneously. Two examples are presented below.

\section{A. High-pressure synthesis and crystal structure of $\mathrm{FeN}$}

Iron nitrides were discovered in the mid-19th century and have been studied intensively since then due to their refractory properties and potential applications as magnetic recording materials. In addition, due to the abundance of nitrogen deeper in Earth's regions, assuming iron nitrides as components of the Earth's core can lead to an improved description of the core's elastic properties. 17,18

Until recently, the iron-nitrogen system was only known for its iron-rich phases $\alpha^{\prime \prime}-\mathrm{Fe}_{16} \mathrm{~N}_{2}, \alpha^{\prime}-\mathrm{Fe}_{8} \mathrm{~N}, \gamma^{\prime}-\mathrm{Fe}_{4} \mathrm{~N}, \varepsilon-\mathrm{Fe}_{3} \mathrm{~N}_{1-\mathrm{x}}, \beta-\mathrm{Fe}_{7} \mathrm{~N}_{3}$, and $\zeta-\mathrm{Fe}_{2} \mathrm{~N}$. ${ }^{19}$ The application of pressure leads to the formation of nitrogen-rich compounds with potentially high hardness and high 
energy density. ${ }^{20-24}$ In particular, very recently, $\mathrm{Fe}_{3} \mathrm{~N}_{2}, \mathrm{FeN}, \mathrm{FeN}_{2}$, and $\mathrm{FeN}_{4}$ were synthesized due to high-pressure high-temperature reactions between iron and nitrogen.

In this study, we have synthesized NiAs-type $\mathrm{FeN}^{25,26}$ and characterized its crystal structure at high-pressure and high-temperature conditions using the scXRD/dsLH setup described above. Three pieces of $\mathrm{Fe}$ were placed into a BX90 $\mathrm{DAC}^{28}$ equipped with $250-\mu \mathrm{m}$ diamond anvils. The cell was then loaded with $\mathrm{N}_{2}$, serving as both a pressure-transmitting medium and reaction agent. A small ruby ball was placed in the pressure chamber for pressure determination. The cell was pressurized to about $50 \mathrm{GPa}$. Laser heating at $1900(200) \mathrm{K}$ provoked a chemical reaction between iron and nitrogen resulting in the synthesis of iron nitride $\mathrm{FeN}$ as single crystals.

The scXRD data were collected both during and after laser heating. In each experiment, we managed to collect 36 unique reflections with intensities $I>3 \sigma(I)$, which enabled the refinement of anisotropic displacement parameters. As expected, the unit cell parameters obtained during and after laser heating $[V=29.853(7)$ and 28.86(4) $\AA^{3}$, respectively] differ due to the thermal expansion of the crystal. The thermal parameters acquired during laser heating are about twice as large as those at room temperature (Table I and Fig. 5). The difference in the quality of the structural refinements for the room- $\left(R_{1}=0.0594 / w R_{2}=0.0692\right)$ and hightemperature data $\left(R_{1}=0.0772 / w R_{2}=0.0793\right)$ is insignificant, proving the technical feasibility of scXRD for in situ studies of materials at high pressures and simultaneous high temperatures in laser-heated DACs.

\section{B. High-pressure synthesis and crystal structure of $\mathrm{CrN}$}

Chromium nitrides are an attractive class of materials with a number of excellent physical and chemical properties. For example, chromium nitride $\mathrm{CrN}$ with a cubic rock salt structure plays a

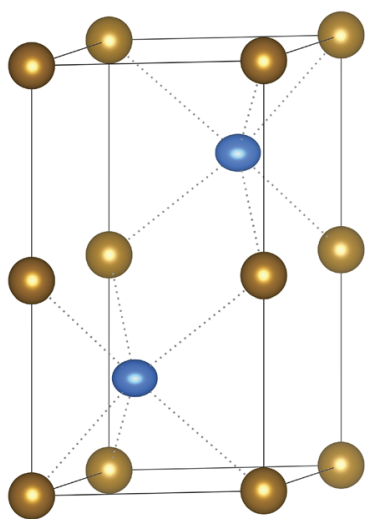

(a)

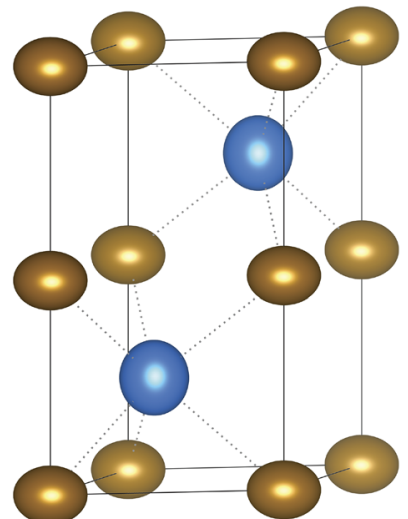

(b)
FIG. 5. Crystal structure of FeN at $293 \mathrm{~K}$ (a) and $1900 \mathrm{~K}$ (b). Orange and blue balls show the positions of $\mathrm{Fe}$ and $\mathrm{N}$ atoms, respectively. Thermal ellipsoids are shown at the $90 \%$ probability level.

fundamental role in the steel hardening process ${ }^{29}$ and is often used as a component in advanced coating systems. ${ }^{30}$

Theoretically predicted nitrogen-rich chromium nitrides $\mathrm{CrN}_{2}$ and $\mathrm{CrN}_{4}$ were suggested to become thermodynamically stable at pressures above 7 and $15 \mathrm{GPa}$, respectively, ${ }^{31,32}$ and are expected to be superhard compounds. ${ }^{31,32}$ The high-pressure reaction between chromium and nitrogen, studied by Hasegawa and Yagi at $10 \mathrm{GPa}$ and $1800 \mathrm{~K}$ in a laser-heated DAC, yielded $\mathrm{CrN}$ as the only product. ${ }^{33}$ Here, we studied the same reaction but at a pressure of $47 \mathrm{GPa}$ in order to search for predicted nitrogen-rich chromium nitrides. $^{31,32}$

A piece of $\mathrm{Cr}$ was loaded into a BX90 DAC equipped with $250 \mu \mathrm{m}$ Boehler-Almax type diamond anvils. The cell was loaded with $\mathrm{N}_{2}$ as pressure-transmitting medium and reaction agent.

TABLE I. Details of crystal structural refinements of $\mathrm{FeN}$ and $\mathrm{CrN}$.

\begin{tabular}{lcccc}
\hline \hline & FeN & FeN & CrN & CrN \\
\hline Pressure $(\mathrm{GPa})$ & 49.6 & 49.6 & 47 & 47 \\
Temperature $(\mathrm{K})$ & 293 & $1900(200)$ & 293 & $1300(200)$ \\
Symmetry & $P 6_{3} / m m c$ & $P 6_{3} / m m c$ & $F m \overline{3} m$ & $F m \overline{3} m$ \\
Lattice parameters & & & & \\
$a=b(\AA)$ & $2.6299(11)$ & $2.6689(4)$ & $3.960(6)$ & $3.9819(5)$ \\
$c(\AA)$ & $4.819(7)$ & $4.8394(6)$ & $3.960(6)$ & $3.9819(5)$ \\
$V\left(\AA^{3}\right)$ & $28.86(4)$ & $29.853(7)$ & $62.10(15)$ & $63.135(14)$ \\
$Z$ & 2 & 2 & 4 & 4 \\
Refinement & & & & \\
No. of measured, independent, & $154 / 40 / 36$ & $112 / 47 / 36$ & $67 / 20 / 19$ & $59 / 18 / 16$ \\
and observed reflections & $0.0594 / 0.0692$ & $0.0772 / 0.0840$ & $0.0886 / 0.0870$ & $0.0705 / 0.1061$ \\
$R_{1} / w R_{2}$ & $0.0116(10)$ & $0.0247(16)$ & $0.0081(15)$ & $0.036(7)$ \\
$U_{\text {iso }}($ metal $)\left(\AA^{2}\right)$ & $0.014(4)$ & $0.026(4)$ & $0.004(3)$ & $0.030(6)$ \\
$U_{\text {iso }}(\mathrm{N})\left(\AA^{2}\right)$ & & &
\end{tabular}




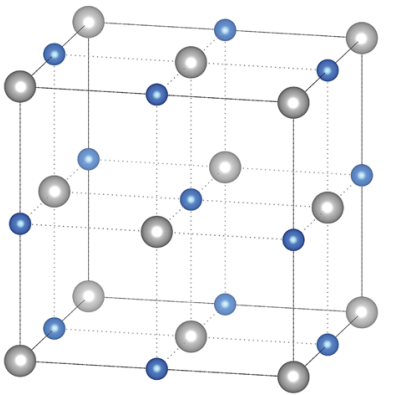

(a)

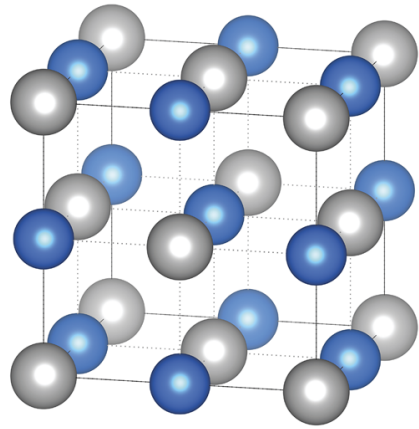

(b)
FIG. 6. The crystal structure of $\mathrm{CrN}$ at $293 \mathrm{~K}$ (a) and $1300 \mathrm{~K}$ (b). Blue and gray balls show the positions of $\mathrm{Cr}$ and $\mathrm{N}$ atoms, respectively. Thermal ellipsoids are shown at the $90 \%$ probability level.

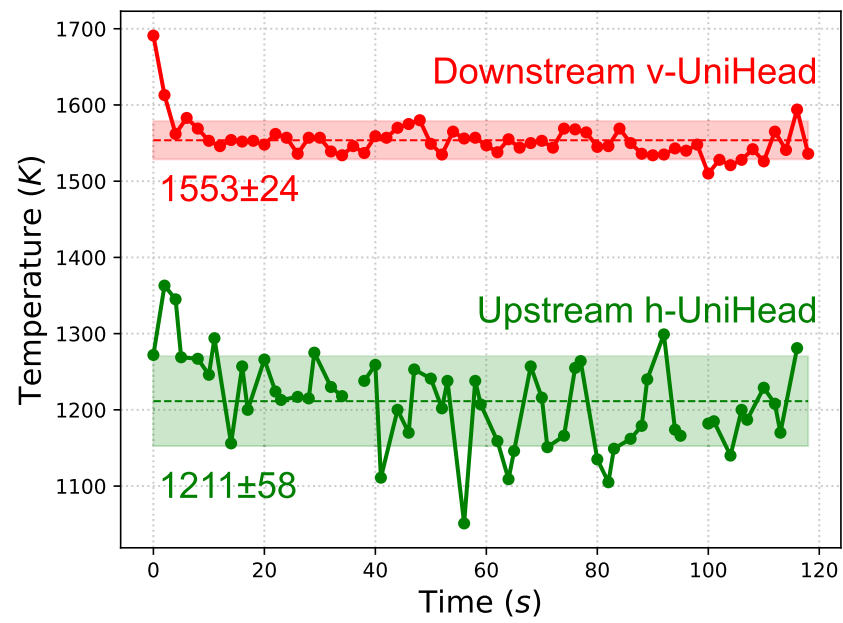

FIG. 7. Time-dependence of the temperature during laser heating and simultaneous scXRD data collection for CrN. Green dots designate the upstream side temperature, and red dots designate the downstream side temperature. Solid lines connecting the dots are guides for the eye. Dashed lines show the mean temperature values for each side over the entire rotation. Shaded areas represent the margins of one standard deviation.

Laser-heating of $\mathrm{Cr}$ in nitrogen to $1300 \mathrm{~K}$ at $47 \mathrm{GPa}$ resulted in the formation of $\mathrm{CrN}$, the same phase, which was observed in Ref. 33 (Table I). The predicted $\mathrm{CrN}_{2}$ and $\mathrm{CrN}_{4}$ phases were not detected.

The CrN crystal showed thermal expansion upon laser heating [the unit cell volumes $V=63.135(14)$ and $62.10(15) \AA^{3}$ were obtained during and after laser heating, respectively]. The thermal parameters acquired during laser heating are larger than those at ambient temperature (Table I and Fig. 6). As shown in Fig. 7, no significant variations in temperature were observed during the entire data collection.

\section{CONCLUSIONS}

We have developed the first dedicated single-crystal X-ray diffractometer coupled with double-sided laser heating system. The scXRD/dsLH setup encompasses all the components required for scXRD data collection and simultaneous double-sided laser heating in a single module which can be mounted/unmounted on the General Purpose experiment of beamline P02.2 (ECB) at PETRA III within a few hours. The alignment of the optical components and preparation of the beamline takes approximately one working day. The setup has been tested in the course of several high-pressure high-temperature experiments aimed at studying chemical reactions between nitrogen and two metals, iron and chromium. It enabled to obtain reliable results in the structure solution and refinement of iron and chromium nitrides.

\section{ACKNOWLEDGMENTS}

N.D. and L.D. thank the Federal Ministry of Education and Research, Germany (BMBF, Grant No. 5K16WC1) and the Deutsche Forschungsgemeinschaft (DFG Project Nos. DU 954-11/1, DU 3939/2, and DU 393-13/1) for financial support. Portions of this research were carried out at the light source PETRA III at DESY, a member of the Helmholtz Association (HGF). The research leading to this result has been supported by the project CALIPSOplus under the Grant Agreement No. 730872 from the EU Framework Programme for Research and Innovation HORIZON 2020.

\section{REFERENCES}

${ }^{1}$ R. L. Mills, D. H. Liebenberg, J. C. Bronson, and L. C. Schmidt, Rev. Sci. Instrum. 51, 891 (1980).

${ }^{2}$ T. Yagi, H. Yusa, and M. Yamakata, Rev. Sci. Instrum. 67, 2981 (1996).

${ }^{3}$ T. Kenichi, P. C. Sahu, K. Yoshiyasu, and T. Yasuo, Rev. Sci. Instrum. 72, 3873 (2001).

${ }^{4}$ B. Couzinet, N. Dahan, G. Hamel, and J.-C. Chervin, High Pressure Res. 23, 409 (2003).

${ }^{\mathbf{5}}$ M. Rivers, V. Prakapenka, A. Kubo, C. Pullins, C. Holl, and S. Jacobsen, High Pressure Res. 28, 273 (2008).

${ }^{6}$ A. Kurnosov, I. Kantor, T. Boffa-Ballaran, S. Lindhardt, L. Dubrovinsky, A. Kuznetsov, and B. H. Zehnder, Rev. Sci. Instrum. 79, 045110 (2008).

${ }^{7}$ R. Boehler, Rev. Sci. Instrum. 77, 115103 (2006).

${ }^{8}$ G. Shen, M. L. Rivers, Y. Wang, and S. R. Sutton, Rev. Sci. Instrum. 72, 1273 (2001).

${ }^{9}$ E. Schultz, M. Mezouar, W. Crichton, S. Bauchau, G. Blattmann, D. Andrault, G. Fiquet, R. Boehler, N. Rambert, B. Sitaud, and P. Loubeyre, High Pressure Res. 25, 71 (2005).

${ }^{10}$ V. B. Prakapenka, A. Kubo, A. Kuznetsov, A. Laskin, O. Shkurikhin, P. Dera, M. L. Rivers, and S. R. Sutton, High Pressure Res. 28, 225 (2008).

${ }^{11}$ R. Boehler, H. G. Musshoff, R. Ditz, G. Aquilanti, and A. Trapananti, Rev. Sci. Instrum. 80, 045103 (2009).

${ }^{12}$ I. Kupenko, L. Dubrovinsky, N. Dubrovinskaia, C. McCammon, K. Glazyrin, E. Bykova, T. Boffa Ballaran, R. Sinmyo, A. I. Chumakov, V. Potapkin, I. Kantor, R. Rüffer, M. Hanfland, W. Crichton, and M. Merlini, Rev. Sci. Instrum. 83, 124501 (2012).

${ }^{13}$ H. P. Liermann, Z. Konôpková, W. Morgenroth, K. Glazyrin, J. Bednarčik, E. E. McBride, S. Petitgirard, J. T. Delitz, M. Wendt, Y. Bican, A. Ehnes, I. Schwark, A. Rothkirch, M. Tischer, J. Heuer, H. Schulte-Schrepping, T. Kracht, and H. Franz, J. Synchrotron Radiat. 22, 908 (2015).

${ }^{14}$ G. Aprilis, C. Strohm, I. Kupenko, S. Linhardt, A. Laskin, D. M. Vasiukov, V. Cerantola, E. G. Koemets, C. McCammon, A. Kurnosov, A. I. Chumakov, R. Rüffer, N. Dubrovinskaia, and L. Dubrovinsky, Rev. Sci. Instrum. 88, 084501 (2017).

${ }^{15}$ G. Shen, L. Wang, R. Ferry, H. Mao, and R. J. Hemley, J. Phys.: Conf. Ser. 215, 012191 (2010).

${ }^{16} \mathrm{M}$. Bykov, "Structural aspects of pressure- and temperature-induced phase transitions in low-dimensional systems," Doctoral thesis (University of Bayreuth, 2015). 
${ }^{17}$ J. F. Adler, J. Geophys. Res. 110, B01203, https://doi.org/10.1029/2004jb003103 (2005).

${ }^{18}$ S. Minobe, Y. Nakajima, K. Hirose, and Y. Ohishi, Geophys. Res. Lett. 42, 5206, https://doi.org/10.1002/2015gl064496 (2015).

${ }^{19} \mathrm{P}$. Höhn and R. Niewa, Handbook of Solid State Chemistry (Wiley-VCH Verlag GmbH \& Co. KGaA, Weinheim, Germany, 2017), pp. 251-359.

${ }^{20}$ D. Laniel, G. Weck, G. Gaiffe, G. Garbarino, and P. Loubeyre, J. Phys. Chem. Lett. 9, 1600 (2018).

${ }^{21}$ B. A. Steele, E. Stavrou, J. C. Crowhurst, J. M. Zaug, V. B. Prakapenka, and I. I. Oleynik, Chem. Mater. 29, 735 (2017).

${ }^{22}$ M. Bykov, E. Bykova, E. Koemets, T. Fedotenko, G. Aprilis, K. Glazyrin, H.-P. Liermann, A. V. Ponomareva, J. Tidholm, F. Tasnádi, I. A. Abrikosov, N. Dubrovinskaia, and L. Dubrovinsky, Angew. Chem., Int. Ed. 57, 9048 (2018).

${ }^{23}$ W. Sun, A. Holder, B. Orvañanos, E. Arca, A. Zakutayev, S. Lany, and G. Ceder, Chem. Mater. 29, 6936 (2017).

${ }^{24}$ M. Bykov, S. Chariton, H. Fei, T. Fedotenko, G. Aprilis, A. V. Ponomareva, F. Tasnádi, I. A. Abrikosov, B. Merle, P. Feldner, S. Vogel, W. Schnick,

V. B. Prakapenka, E. Greenberg, M. Hanfland, A. Pakhomova, H.-P. Liermann, T. Katsura, N. Dubrovinskaia, and L. Dubrovinsky, Nat. Commun. 10, 2994 (2019).
${ }^{25}$ M. Bykov, E. Bykova, G. Aprilis, K. Glazyrin, E. Koemets, I. Chuvashova, I. Kupenko, C. McCammon, M. Mezouar, V. Prakapenka, H.-P. Liermann, F. Tasnádi, A. V. Ponomareva, I. A. Abrikosov, N. Dubrovinskaia, and L. Dubrovinsky, Nat. Commun. 9, 2756 (2018).

${ }^{26}$ W. P. Clark, S. Steinberg, R. Dronskowski, C. McCammon, I. Kupenko, M. Bykov, L. Dubrovinsky, L. G. Akselrud, U. Schwarz, and R. Niewa, Angew. Chem., Int. Ed. 56, 7302 (2017).

${ }^{27}$ M. Bykov, S. Khandarkhaeva, T. Fedotenko, P. Sedmak, N. Dubrovinskaia, and L. Dubrovinsky, Acta Crystallogr., Sect. E: Crystallogr. Commun. 74, 1392 (2018).

${ }^{28}$ I. Kantor, V. Prakapenka, A. Kantor, P. Dera, A. Kurnosov, S. Sinogeikin, N. Dubrovinskaia, and L. Dubrovinsky, Rev. Sci. Instrum. 83, 125102 (2012).

${ }^{29}$ M. Widenmeyer, E. Meissner, A. Senyshyn, and R. Niewa, Z. Anorg. Allg. Chem. 640, 2801 (2014).

${ }^{30}$ F. Rivadulla, M. Bañobre-López, C. X. Quintela, A. Piñeiro, V. Pardo, D. Baldomir, M. A. López-Quintela, J. Rivas, C. A. Ramos, H. Salva, J.-S. Zhou, and J. B. Goodenough, Nat. Mater. 8, 947 (2009).

${ }^{31}$ A. G. Kvashnin, A. R. Oganov, A. I. Samtsevich, and Z. Allahyari, J. Phys. Chem. Lett. 8, 755 (2017).

${ }^{32}$ Z. Zhao, K. Bao, F. Tian, D. Duan, B. Liu, and T. Cui, Phys. Rev. B 93, 214104 (2016).

${ }^{33}$ M. Hasegawa and T. Yagi, J. Alloys Compd. 403, 131 (2005). 